\title{
Energy Efficiency Opposition-Based Learning and Brain Storm Optimization for VNF-SC Deployment in IoT
}

\author{
Hejun Xuan $\mathbb{D}^{1},{ }^{1}$ Xuelin Zhao, ${ }^{1}$ Zhenghui Liu, ${ }^{1,2}$ Jianwei Fan, ${ }^{1,2}$ and Yanling $\mathrm{Li}^{1,2}$ \\ ${ }^{1}$ School of Computer and Information Technology, Xinyang Normal University, Xinyang 464000, China \\ ${ }^{2}$ Henan Key Lab. of Analysis and Application of Education Big Data, Xinyang Normal University, Xinyang 464000, China \\ Correspondence should be addressed to Hejun Xuan; xuanhejun0896@xynu.edu.cn
}

Received 25 November 2020; Revised 30 December 2020; Accepted 3 February 2021; Published 13 February 2021

Academic Editor: Pei-Wei Tsai

Copyright (C) 2021 Hejun Xuan et al. This is an open access article distributed under the Creative Commons Attribution License, which permits unrestricted use, distribution, and reproduction in any medium, provided the original work is properly cited.

\begin{abstract}
Network Function Virtualization (NFV) can provide the resource according to the request and can improve the flexibility of the network. It has become the key technology of the Internet of Things (IoT). Resource scheduling for the virtual network function service chain (VNF-SC) is the key issue of the NFV. Energy consumption is an important indicator for the IoT; we take the energy consumption into the objective and define a novel objective to satisfying different objectives of the decision-maker. Due to the complexity of VNF-SC deployment problem, through taking into consideration of the heterogeneity of nodes (each node only can provide some specific VNFs), and the limitation of resources in each node, a novel optimal model is constructed to define the problem of VNF-SC deployment problem. To solve the optimization model effectively, a weighted center oppositionbased learning is introduced to brainstorm optimization to find the optimal solution (OBLBSO). To show the efficiency of the proposed algorithm, numerous of simulation experiments have been conducted. Experimental results indicate that OBLBSO can improve the accuracy of the solution than compared algorithm.
\end{abstract}

\section{Introduction}

Internet of Things (IoT) is turning into the future generation of wireless network communication technology and sensor networks. IoT devices are cost-effective, so distributed expensive devoted spectrum to the IoT would be inefficient. Therefore, the problem of resource allocation that satisfies the constraints of network operation is particularly important. The IoT can work as an additional layer on the basic network of any communication technology [1,2]. The spectrum access problem solution for the IoT network is a combination of low-cost networks that can be combined. The use of a variety of network technologies is to serve the traffic of the IoT [3]. Eternal virtualization has many advantages, which can improve VONs with different widely used topologies. In addition, it can enable VONs to share physical network explorer between different users and applications, reduce physical resource management, and provide simple spectrum allocation [4-7]. However, how to map a large number of von of different topologies to the physical network while achiev- ing certain goals, such as energy consumption, blocking rate, and network performance, is a challenge $[8,9]$. In recent years, there has been a lot of research focused on the VONs mapping problem and related issues [10]. Virtualization (NFV) with Network Function Virtualization makes it possible to manage mobility within the infrastructure such as the Service Function Chaining (SFC). With the change of Network load and node and link state in the infrastructure, the migration of Virtual Network Function (VNF) in SFC can improve the utilization rate of underlying resources and meet the requirements of different slices for delay. In addition, the flexible arrangement of VNF also provides a favorable condition for saving system energy consumption. Under the mechanism of VNF sharing, VNF migrates servers with low resource utilization, and shutdown of the corresponding server can achieve the purpose of reducing energy consumption $[11,12]$.

In this work, we studied the spectrum allocation of VON mapping service chain (VNF-SC) in the IoT. Different from the previous work, we have studied the IoT, that is, each node 
can only provide some specific VNFs, and the system resources of all nodes are limited. The major contributions of this study are summarized as follows:

(i) Energy consumption is an important indicator for the IoT; we take the energy consumption into the objective and define a novel objective to satisfying different objectives of the decision-maker

(ii) Due to the complexity of VNF-SC deployment problem, through taking into consideration of the heterogeneity of nodes (each node only can provide some specific VNFs), and the limitation of resources in each node, a novel optimal model is constructed to define the problem of VNF-SC deployment problem

(iii) Since a large number of variables are in the optimal model, it has numerous local optimal solutions. For the sake of jumping out the local optimal, a weighted center opposition learning strategy is proposed. Based on this, an improved brain storm optimization algorithm, which can improve the accuracy of the solution, is proposed. Experimental results demonstrate that the proposed algorithm can obtain a better solution than the compared algorithm

\section{Related Work}

Last few years, some studies have been conducted on network scheduling issues using VNF-scs, mainly focusing on service link routing and VNF deployment issues (e.g., [13-15]). Literature [16] minimizes the sum of the three costs of cloud resource cost, bandwidth cost, and reconstruction cost. The characteristic of reconstruction cost is the loss of revenue generated by network operators due to bit loss. Considering the constraints of flexible optical network and DC capacity, an effective algorithm based on noncooperative mixed strategy game is proposed [17]. In order to solve the relatively long setup delay and complicated network control problems, we designed a configuration framework with resource predeployment to solve the above problems [18]. The proposed game model enables tenants to compete for VNF-SC supply services based on the incentives of income and service quality, so it can encourage tenants to choose more reasonable supply solutions. In order to meet the needs of users and maximize the benefits of suppliers, a VNF deployment algorithm based on eigenvalue decomposition is proposed [19]. So far, most of the literatures, such as literature [20], have studied the issues related to the migration of virtual machines under a certain migration trigger time, but such a migration strategy does not consider multiple SFC business scenarios at the same time. Literature [21] studies the VNF migration problem under the one-to-one mapping relationship between service functional chain VNF and node VNF instances. In the shared state of VNF instances, if the performance of a slice fails to meet user requirements, the current research cannot formulate an effective strategy to achieve VNF migration. Literature [22] proposed a VNF migration algorithm based on MDP theory to deal with the constantly changing workload, and its migration strategy was aimed at minimizing energy consumption and reconfiguration cost caused by VNF migration. Literature [23] established a cost model and proposed a greedy algorithm to optimize the migration of VNF, but this scheme can only solve the problem of resource allocation in a single scheduling cycle. Literature [24] makes backup for the whole SFC, which increases the resource overhead. In literature [25], a reliability perception method combining VNF deployment and routing optimization is proposed, which adopts a backup sharing method to reduce resource consumption. While using the backup mechanism to improve reliability, the link length of SFC is increased, and the end-to-end delay of SFC is increased to a certain extent. In literature [26], no backup mechanism was adopted. PageRank thought was adopted when deploying VNF, and reliability and delay were considered at the same time. However, when deploying VNF, the source node and destination node were not considered, so the delay was increased. Literature [27] did not adopt the backup mechanism and proposed the SFC mapping algorithm based on queue awareness to improve the stability and reliability of the network, which took into account the source nodes and destination nodes. Literature [27] does not constrain VNF types but assumes that each physical node can carry any type of VNF. Literature [27] pointed out that adjacent VNF in the same SFC can be aggregated, that is, deployed on the same physical node, but it did not provide a specific polymerization method.

\section{Problem Description and Modeling}

3.1. Network and VNF-SC Description. $G(V, E)$ is an undirected graph, and we use it to denote an IoT, where $V=\left\{v_{1}, v_{2}, \cdots, v_{N_{V}}\right\}$ is nodes set in the network. $N_{V}$ denotes the number of nodes, $v_{i}$ is the $i$ th optical node. $E=\left\{l_{i j} \mid v_{i}, v_{j} \in V\right\}$ denotes the set of links. $l_{i j}$ represents the link between $v_{i}$ and $v_{j} . N_{E}$ represents the number of links. Each link has $N_{F}$ frequency slots (FSs), and the indexes of FSs on each link are $1,2, \cdots, N_{F}$.

Each VNF-SC is a task. Now, we have a set of VNF-SCs, denoted by $T=\left\{T_{1}, T_{2}, \cdots, T_{N_{T}}\right\}$, where $N_{T}$ represents the tasks (VNF-SCs) number, and $T_{k}$ is the $k$ th VNF-SC. $T_{k}$ can be described as $T_{k}=\left(s_{k}, d_{k}, V N F_{k}^{T}, b_{k}\right)$, where $s_{k}$ and $d_{k}$ represent source node and destination node, and $V N F_{k}^{T}=$ $\left\{V N F_{k_{1}}, V N F_{k_{2}}, \cdots, V N F_{k_{M_{k}}}\right\}$ is the of virtual network functions to be realized in $T_{k}$, and $M_{k}$ denotes the number of virtual network functions in $V N F_{k}^{T}$, i.e., some nodes should be chosen in the selected path to realize these virtual network functions. $b_{k}=\left(b_{k}^{0}, b_{k}^{1}, \cdots, b_{k}^{M_{k}}\right)$ represents the frequency slots numbers of $T_{k}$ required, where $b_{k}^{0}$ is the original number of frequency slots occupied by $T_{k}$. What is more, $\forall V N F_{k}^{T} \subseteq$ $\operatorname{VNF}\left(1 \leq k \leq N_{T}\right)$.

\section{Energy Model}

The total energy consumption of telecommunications networks supporting virtual network functions consists of two 
parts. Let $N_{f}^{v}$ denotes the number of virtual network functions deployed on node $v$ :

$$
N_{f}^{v}=\left\lceil\frac{\sum_{r \in R} z_{r, f}^{v} \cdot b_{r}}{c t_{f}}\right\rceil
$$

Since server energy consumption is positively correlated with CPU utilization, the total energy consumption of VNF $f$ deployed on node $v$ can be derived as

$$
p_{f}^{v}=\frac{p_{h}^{s}-p_{b}^{s}}{C_{v}} \cdot c r_{f} \cdot \frac{\sum_{r \in R} z_{r, f}^{v} \cdot b_{r}}{c t_{f}}, \forall v \in V, f \in F .
$$

Among them, $p_{b}^{s}$ represents the startup energy consumption of the node $s$, and $p_{h}^{s}$ represents the peak load energy consumption of the node. Let $p_{v}$ denote the energy consumption of node $s$ :

$$
p_{v}=p_{b}^{s} \cdot \min \left\{1, \sum_{f \in F} \sum_{r \in R} z_{r, f}^{v}\right\}+\sum_{f \in F} p_{f}^{v}
$$

where $\min \left\{1, \sum_{f \in F} \sum_{r \in R} z_{r, f}^{v}\right\} \in\{0,1\}$ means some VNF instance is deployed on the node $v$. When it is equal to 1 , the node $v$ must be opened to carry the VNF instance. Represents the link bandwidth utilization, let $U_{l}$ denote the bandwidth utilization of link $l$ :

$$
U_{l}=\frac{\sum_{r \in R} w_{r}^{u f v g} \cdot y_{r}^{u v l} \cdot b_{r}}{C_{l}}, \forall l \in L, f \in F
$$

The energy consumption of the link can be calculated as follows:

$$
p_{l}=p_{b}^{l} \cdot \min \left\{1, \sum_{f \in F} \sum_{r \in R} w_{r}^{u f v g} \cdot y_{r}^{u v l}\right\}+\left(p_{h}^{l}-p_{b}^{l}\right) \cdot U_{l} .
$$

Among them, $p_{b}^{l}$ is the start-up energy consumption of the link $l$, and $p_{b}^{l}$ is the peak load energy consumption of the link. $\min \left\{1, \sum_{f \in F} \sum_{r \in R} w_{r}^{u f v g} \cdot y_{r}^{u v l}\right\} \in\{0,1\}$ represents the on/off status of the dollar link $l$; when it is equal to 1 , the link $l$ must be powered on to the VNF-SC. Therefore, the total energy consumption of NFV-SC can be calculated as

$$
p_{\text {total }}=\sum_{v \in V} p_{v}+\sum_{l \in L} p_{l}
$$

The other two objectives are given in the [28]. The objective function is expressed by

$$
\min H=\min \left\{\alpha_{1} p_{\text {total }}+\alpha_{2} f_{2}+\alpha_{3} f_{3}\right\} \text {, }
$$

where $f_{1}$ and $f_{2}$ denotes the maximum index of used frequency slots and ratio of resource used. Some constraint conditions should be satisfied. These constraint conditions are given in our previous paper [29]. To solve the optimization model, we propose an improved brain storm optimization algorithm, and the algorithm will be described in the following sections.

\section{Proposed Algorithm}

5.1. Bounding-Box Determines the Search Area. The basic idea of the Bounding box algorithm is to determine the possible Bounding rectangular regions of unknown nodes by measuring their distance from unknown nodes and using their distance. Finally, the center of masses of all Bounding regions is taken as the estimated position of unknown nodes. Although the algorithm is simple and easy to implement, its positioning accuracy is relatively low. References [30] put forward individual Bounding box initiative-inspired optimization algorithm to improve convergence speed and avoid overturning ambiguity in the positioning process. However, due to the impact of ranging errors, the real position of unknown nodes may fall outside the Bounding box region, reducing convergence speed and solving accuracy. To avoid this phenomenon, this paper improves the Bounding box method and determines the search area through multiple signal measurements and compensated measurement distances. The individual heuristic algorithm is initialized in the search area to improve convergence speed and solution accuracy. Assuming that there are $\mathrm{m}$ anchor nodes in the communication range of the unknown node, the coordinates of the unknown node $\left(x_{i}, y_{i}\right)$ satisfy equation (8):

$$
\left\{\begin{array}{l}
x_{i} \in\left[x_{j}-d_{i j}, x_{j}+d_{i j}\right] \\
y_{i} \in\left[y_{j}-d_{i j}, y_{j}+d_{i j}\right]
\end{array} .\right.
$$

5.2. Weighted Center Opposition Learning Strategy. The Opposition based Learning (OBL) strategy was proposed by Tizhoosh in 2005 [31]. It has been widely used in various algorithms, effectively improving the efficiency of solving the global optimum. The basic idea of the reverse learning strategy is as follows: in the search process, the initial position and its reverse position relative to the center are considered simultaneously, so as to enhance the diversity of individual groups and improve the global search capability of the algorithm. Let point $P=\left(p^{1}, p^{2}, \cdots, p^{i}, \cdots, p^{d}\right)$ is a point in $D$ dimensional space, and $p_{\text {min }}^{i} \leq p^{i} \leq p_{\text {max }}^{i}(i=1,2, \cdots, d) \cdot p_{\text {min }}^{i}$ and $p_{\text {max }}^{i}$ are the minimum and maximum value of point $p^{i}$ in dimension $D$, respectively. Then, the opposite point of point $p^{i}$ is

$$
\widehat{p^{i}}=p_{\max }^{i}+p_{\min }^{i}-p^{i}
$$

Literature [32] proposed that the search population takes the mean mixing center as the symmetric center of reverse learning to guide the population evolution. Among them, the mean blending center is determined by the fitness value of the mean value of all individuals and the mean value of some better individuals. As the center of the mixed mean is located in the center of the group, individual positions can be integrated in the search process, which will promote the 
1 Propose problems that need to be solved and gather people with different knowledge backgrounds;

2 These people come up with many solutions based on this problem and following the four principles;

3 Find several parties as owners of the problem and choose from each of these ideas the best solution they think will solve the problem;

4 Select some solutions to come up with more solutions, and the better solution in Step 3 has a greater probability of being selected;

5 Similar to step 3, the problem owner selects several better solutions;

6 Randomly select a solution and use its functions and characteristics as clues to generate more solutions;

7 Let the owner of the problem choose a few better solutions from all the solutions;

8 End up with a solution that's good enough;

Algorithm 1: Brainstorming Process.

group to draw closer to the center in the early stage and accelerate the convergence speed. After convergence, individuals can jump out of the optimal group and, thus, have a higher probability to search for the global optimal solution. Inspired by his ideas, we propose a weighted center reverse learning strategy. The basic idea is that the weight of all individuals in the population is given according to their fitness value, the weighted center of the population is calculated according to the weight and individual position, and the weighted center is taken as the symmetric center of reverse learning. Since fitness values of all individuals are taken into account, the weighted center can better reflect the central trend of the population in the current environment. Therefore, this paper uses the weighted center as the symmetric center of reverse learning to guide population evolution.

The fitness value $J\left(S P_{i}\right)$ of an individual is the value of the objective function. When solving the weight center, the individual weight is calculated as follows:

$$
W_{i}=\frac{1 / J\left(S P_{i}\right)}{\sum_{j=1}^{m}\left(1 / J\left(S P_{j}\right)\right)}
$$

$S P_{i}^{d}$ and $\bar{C}^{d}$, respectively, represent the values of individual $\mathrm{I}$ and the weighted center on dimension $D . \bar{C}^{d}$ can be calculated by

$$
\bar{C}^{d}=\sum_{i=1}^{m}\left(W_{i} \cdot S P_{i}^{d}\right) .
$$

Opposition point position of point $p$ on dimension $D$ with the weighted center as the symmetric point can be calculated by

$$
\bar{p}^{d}=k\left(\bar{C}^{d}-p_{d}\right)+\bar{C}^{d}
$$

where $p_{d}$ is the value of point $p$ on dimension $d, \bar{p}^{d}$ is the value of point $p$ on dimension $D$ after opposite learning by weighted center, and $k \in[0,1]$ is the dynamic learning factor.

\section{Brain Storm Optimization}

Swarm intelligence optimization algorithm is an optimization algorithm inspired by the biological behavior of nature. It is considered as a young and promising swarm intelligence optimization algorithm, which simulates the brainstorm process of human beings in solving problems.

6.1. Brainstorming Process. When we come across a difficult problem that cannot be solved by one person alone, we will gather people with different knowledge backgrounds to brainstorm, and usually, the problem will be solved with a high probability. The specific steps of the brainstorming process are as follows:

In this paper, a weighted center opposition based learning is introduced to brainstorm optimization to find the optimal solution (OBLBSO), the improved Brain Storm Optimization is shown in Algorithm 2.

The brain storm optimization algorithm is a new one which simulates the brain storm conference process design. When solving the static single objective optimization problem, the detailed steps of the algorithm are shown in Algorithm 1. Static single objective brainstorm optimization algorithm mainly consists of three parts: individual clustering in decision space, generating new individuals, and selecting better individuals.

\section{Experiments and Analysis}

In order to verify the effectiveness and effectiveness of the algorithm, experiments are carried out on the NSFNET topology.

\subsection{Parameters Setting}

7.1.1. Network Parameters. There are $N_{v n f}=5$ kinds of VNFs, and each data center can provide 2-5 types of VNFs. Each $\mathrm{VNF}-\mathrm{sc}$ requires at least one $\mathrm{VNF}$, and the required frequency slots meet uniform distribution $[5,10]$. In addition, in $[0.5,1.5]$, the required frequency-to-slot ratio satisfies a uniform distribution after implementing the corresponding VNFs. Each fiber can accommodate 1000 frequency slots, that is, $N_{F}=1000$. In the proposed improved brain storm optimization algorithm, the following parameters are chosen: population size $P_{s}=100$, maximum iterations $G_{\max }=30,000$, $\tau_{1}=\tau_{2}=\tau_{3}=2$.

7.2. Experimental Results. And compared with several other algorithms, the algorithm proposed in the literature [33] (LBA for short) is used for improvement. The second is the LF-LBA algorithm, including minimum priority strategy and LBA algorithm. In addition, we also compared OBLBSO 
$1 \mathrm{~N}$ individuals are randomly generated;

2 The individuals were clustered in the decision space and divided into $G$ clusters;

3 Evaluate individual fitness value;

4 The individuals in each cluster were sorted and the best individuals in each cluster were recorded as the center of the cluster;

$5 \mathrm{~A}$ random number between 0 and 1 is generated randomly. If the random number is less than the preset probability of $p_{1}$, an individual will be generated randomly to replace a cluster center;

6 Randomly generate a number $p_{3}$ between 0 and 1 ;

7 if $p_{3}$ is less than the default probability of $p_{2}$ then

8 Random into a random number between 0 and 1, and randomly choose a cluster;

9 If the random number is less than $p_{4}$, the center of the cluster is selected and a new individual is generated through Gaussian variation;

10 Otherwise, other individuals in the cluster are selected and new individuals are generated by Gaussian variation;

11 else

12 Randomly generate a number between 0 and 1 ;

13 If the random number is less than $p_{5}$, then a new individual is generated based on the center of the two clusters through Gaussian variation. Otherwise, two individuals selected at random based on two clusters will be generated by Gaussian variation;

14 end

15 The newly generated individuals were compared with the existing ones, and the well-preserved individuals were taken as the next generation of new individuals;

16 If a preset maximum number of iterations is reached, stop, or skip to Step 2.

Algorithm 2: VNF-SC mapping algorithm-based OBLBSO.

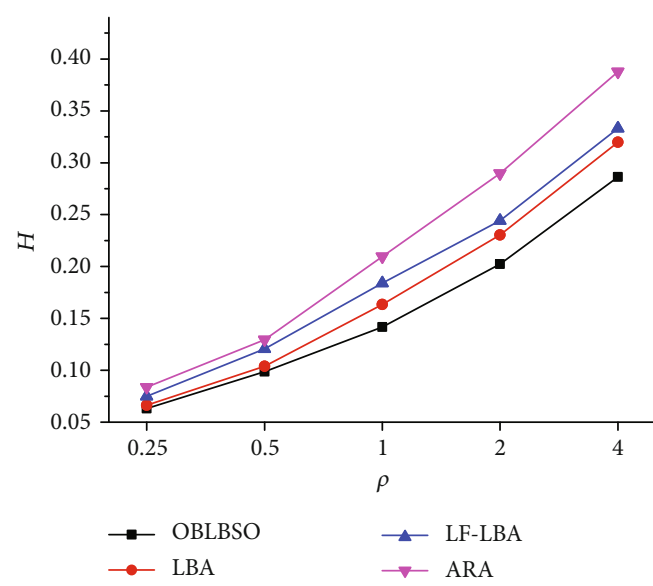

(a) $N_{D}=1 / 3 N_{V}$

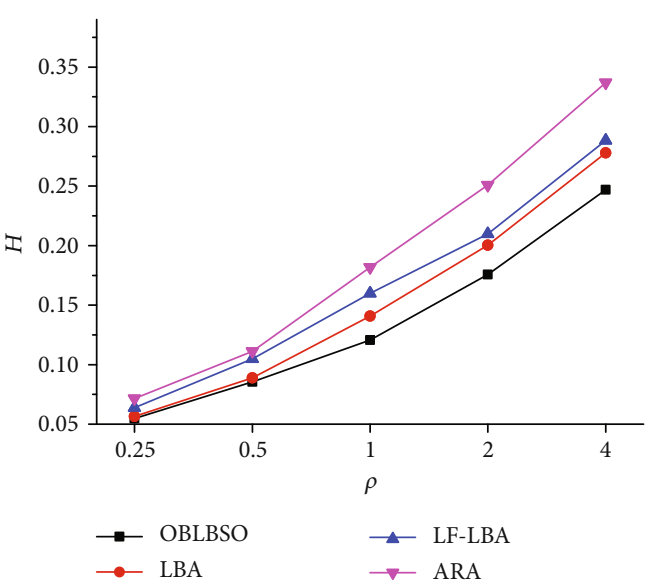

(b) $N_{D}=2 / 3 N_{V}$

FIgURE 1: Experimental results when $\alpha_{1}=1$.

with the ARA (artificial raindrop algorithm, ARA) proposed in the literature [34].

For the sake of verifying the performance of the model and algorithm, two experimental scenarios were carried out. In the first scenario, we fixed the number of target nodes as $N_{D}=N_{V} / 3$ and $N_{D}=2 N_{V} / 3$, i.e., $N_{d}=N_{D} / N_{V}=1 / 3$ and $N_{d}=N_{D} / N_{V}=2 / 3$. The number of destination nodes generated in $\left[N_{V} / 6, N_{V} / 3\right]$ and $\left[N_{V} / 3,2 N_{V} / 3\right]$ randomly. Figure 1 shows the experimental results when $\alpha=1$. Experimental results when $\beta=1$ is shown in Figure 2. Figure 3 shows the experimental results when $\gamma=1$. Experimental results when $\alpha=\beta=\gamma=1 / 3$ is shown in Figure 4 . Number of connection requests are set as $N_{R}=\rho N_{V}\left(N_{V}-1\right)$, and $\rho=0.25,0.5,1$, 2 , and 4 , respectively.

In the second scene, we fixed $\alpha_{1}, \alpha_{2}$ and $\alpha_{3}$ to $\alpha_{1}=\alpha_{2}=$ $\alpha_{3}=1 / 3$. Figure 5 , respectively, shows $\rho=0.25 \mathrm{~s}, \rho=0.5, \rho=$
$1, \rho=2, \rho=4$, and $\rho=8$. The result obtained in the NSFNST topology at the time. In each experiment, set the number of connection requests to $N_{D}=\theta N_{V}$ and select $\theta=$ to be 0.2 , $0.4,0.6,0.8$, and 1 , respectively.

7.3. Experimental Analysis. The experimental results obtained under NSFNET topology are shown in the figure. When $\alpha_{1}, \alpha_{2}$, and $\alpha_{3}$ are selected as 1, 0, 01 , therefore, the objective function is to minimize the maximum use frequency slot index (MIUFS). It can be seen from the experimental that the OBLBSO achieves better results than the other algorithm. Generally speaking, the minimum priority strategy can reduce the maximum index of the frequency slot used. However, VNF dependencies can disable it. Therefore, in some cases, LBA can achieve a better solution than LF-LBA, and in other cases, LF-LBA is a better solution 


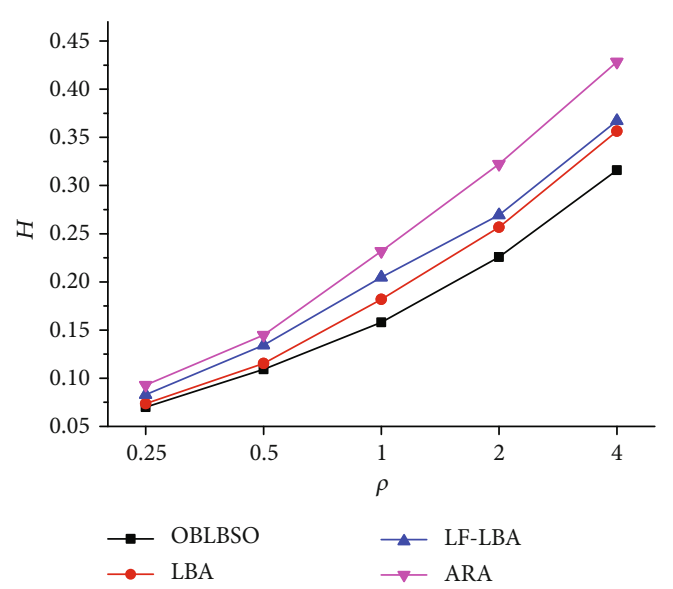

(a) $N_{D}=1 / 3 N_{V}$

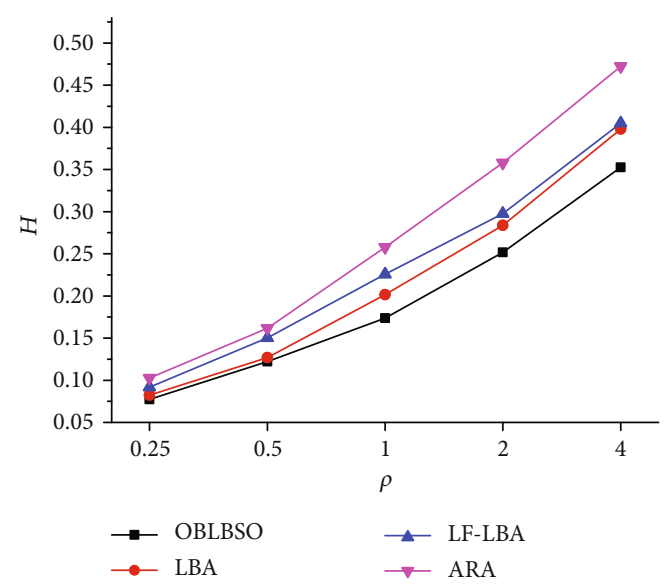

(b) $N_{D}=2 / 3 N_{V}$

FIgURE 2: Experimental results when $\alpha_{2}=1$.

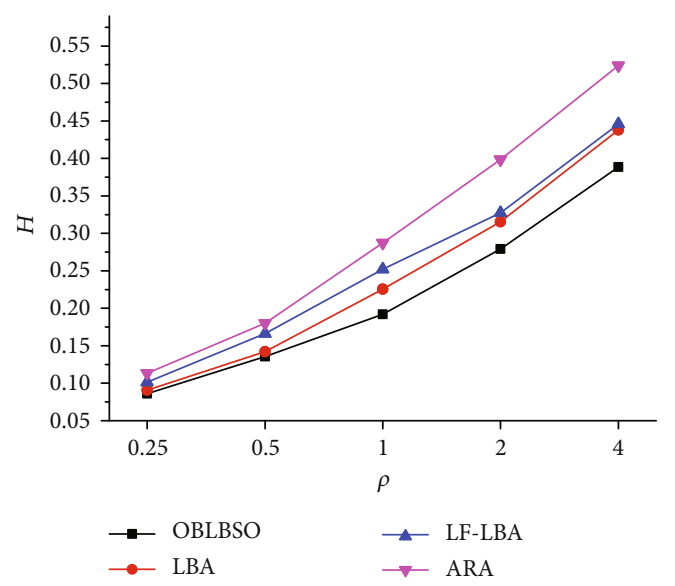

(a) $N_{D}=1 / 3 N_{V}$

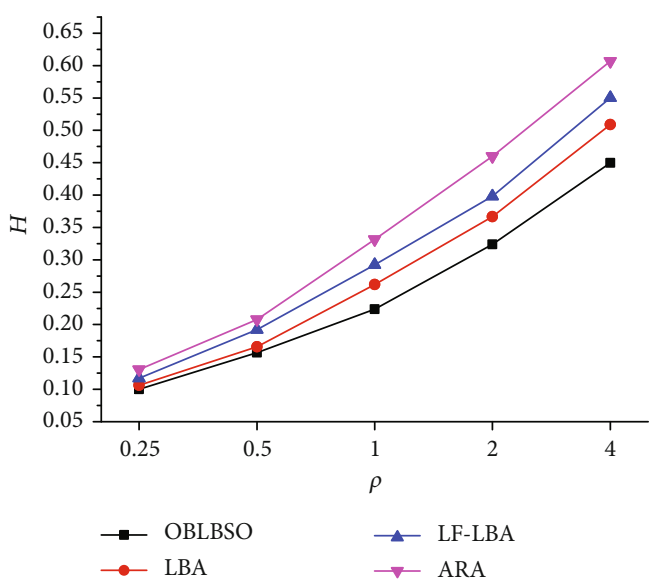

(b) $N_{D}=2 / 3 N_{V}$

FIGURE 3: Experimental results when $\alpha_{3}=1$.

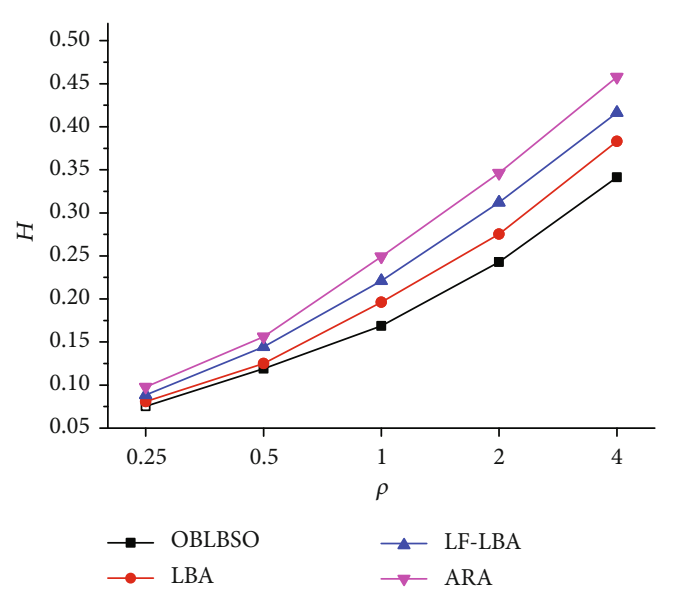

(a) $N_{D}=1 / 3 N_{V}$

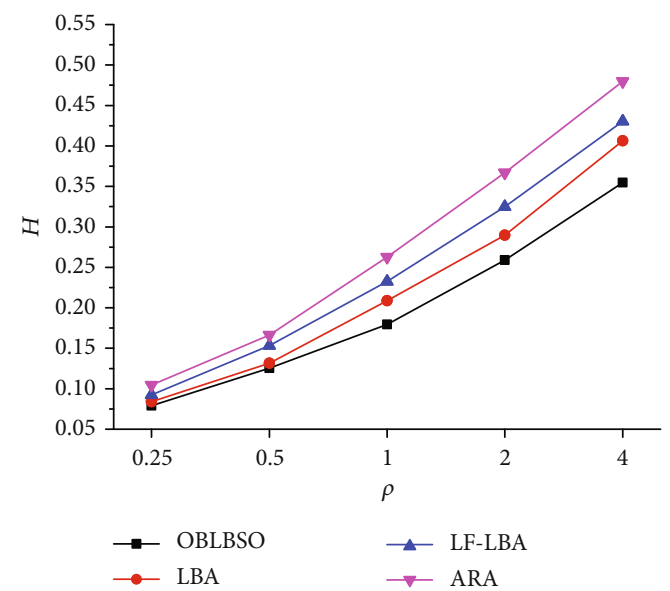

(b) $N_{D}=2 / 3 N_{V}$

FIGURE 4: Experimental results when $\alpha_{1}=\alpha_{2}=\alpha_{3}=1 / 3$. 


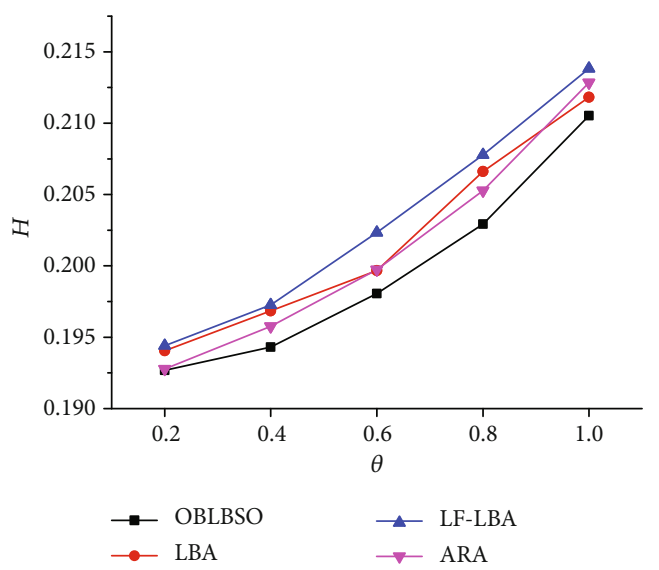

(a) $\rho=0.25$

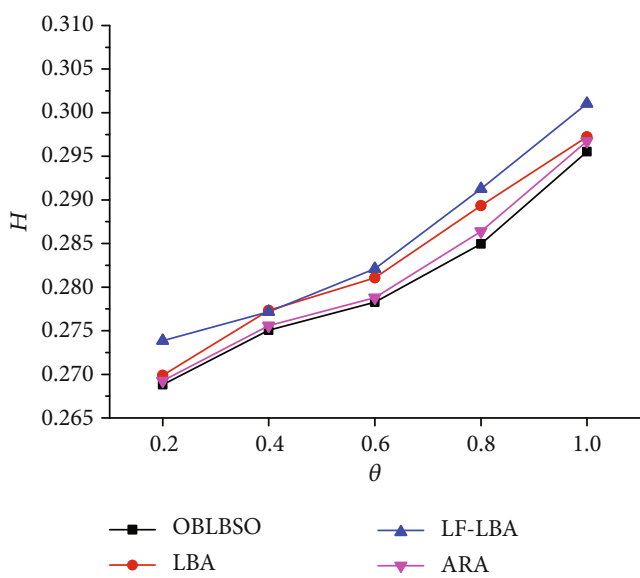

(c) $\rho=1$

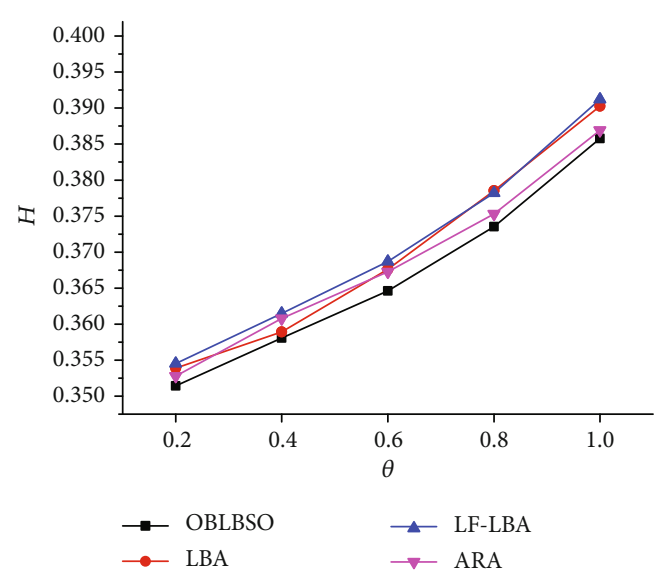

(e) $\rho=4$

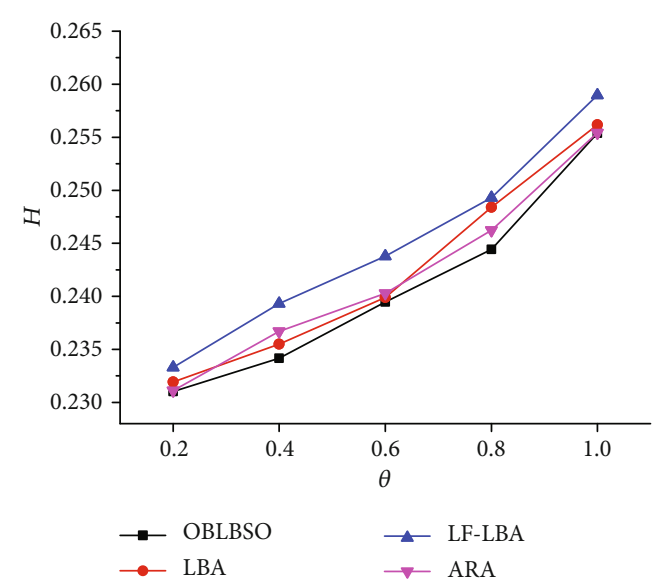

(b) $\rho=0.5$

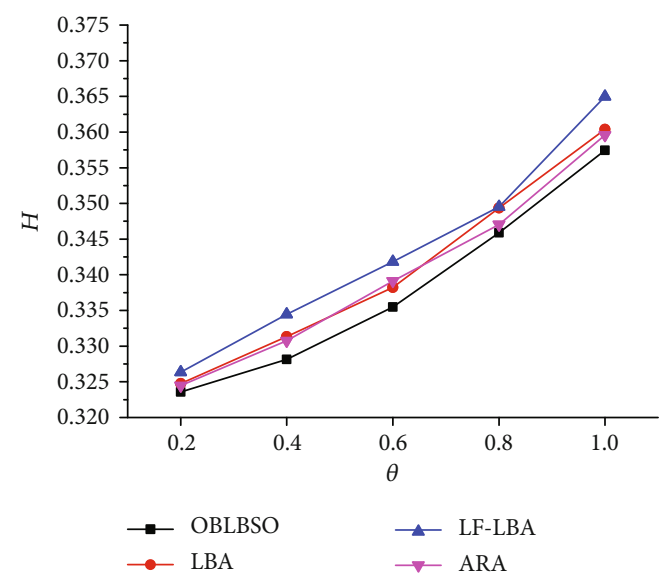

(d) $\rho=2$

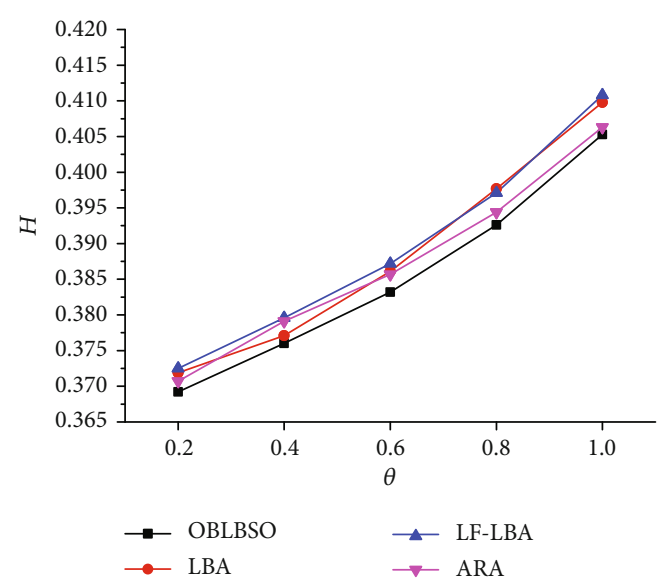

(f) $\rho=8$

Figure 5: Experimental results obtained when $\rho=0.25,0.5,1,2,4,8$.

than LBA. OBLBSO can find the optimal routing and VNFs deployment plan for all VNF-SCs. Therefore, in the three algorithms, OBLBSO can get the optimal solution. When the number of connection requests is $0.25 N_{V}\left(N_{V}-1\right)$, the MIUFS obtained by OBLBSO is $3.7 \%-4.5 \%$ less than the MIUFS obtained by the other algorithms. When the number of connection requests is $2 N_{V}\left(N_{V}-1\right)$, the MIUFS obtained by OBLBSO is $7.9 \%-9.0 \%$ less than the MIUFS obtained by the other algorithms, respectively. In other words, as the number of connection requests increases, OBLBSO can obtain a smaller total power consumption and save more power than other algorithms.

When $\alpha, \beta$, and $\gamma$ are selected as $0,1,0$, the experimental results are shown in Figure 2. Similar to the experimental results in Figure 1, we can also see that the OBLBSO achieves better results than the other algorithms. In addition, based on 
the experimental results, we cannot distinguish between LBA and LF-LBA.

Figure 3 shows the experimental results when $\alpha_{1}, \alpha_{2}$, and $\alpha_{3}$ are selected as 1,0 , and 0 . Figure 4 shows the experimental results at $\alpha_{1}=\alpha_{2}=\alpha_{3}=1 / 3$. It can be seen from the experimental results that the OBLBSO can obtain a better solution than the two other algorithms.

It can be seen from the experimental results that using OBLBSO can get better results than ARA. On this basis, the individual location update strategy has been improved. This algorithm, like the particle swarm algorithm and the $\mathrm{DE}$ algorithm, uses the location of other individuals and their past location information, thereby enhancing the search capability and improving the convergence speed.

\section{Conclusion}

The deployment of VNFs of VNF-SC in flexible optical networks between data centers is studied. In an elastic optical network between data centers, each data center can only provide specific VNFs, and system resources are limited. A mixed integer linear programming model is established, and an improved brainstorming optimization algorithm (OBLBSO) is proposed to solve the model. A simulation experiment was carried out on a widely used network topology.

\section{Data Availability}

All the data can be found in the manuscript.

\section{Conflicts of Interest}

The authors declare that they have no conflicts of interest.

\section{Acknowledgments}

This work was supported by the National Natural Science Foundation of China (No. 62006205, 62002307), Innovation Team Support Plan of University Science and Technology of Henan Province (No. 19IRTSTHN014), Foundation of Henan Educational Committee under Contract (No. 21A520039), and Nanhu Scholars Program for Young Scholars of XYNU, Youth Sustentation Fund of Xinyang Normal University (No.2019-QN-040).

\section{References}

[1] D. Bhamare, R. Jain, M. Samaka, and A. Erbad, "A survey on service function chaining," Journal of Network and Computer Applications, vol. 75, pp. 138-155, 2016.

[2] B. Blanco, I. Taboada, J. Oscar Fajardo, and F. Liberal, "A robust optimization based energy-aware virtual network function placement proposal for small cell $5 \mathrm{~g}$ networks with mobile edge computing capabilities," Mobile Information Systems, vol. 2017, no. 4, pp. 1-14, 2017.

[3] C. Galdamez, R. Pamula, and Z. Ye, "On efficient virtual network function chaining in nfv-based telecommunications networks," Cluster Computing, vol. 22, no. 3, pp. 693-703, 2019.
[4] J. Masahiko, "Virtualization in optical networks: From elastic networking level to sliceable equipment level," in The 10th International Conference on Optical Internet (COIN2012), pp. 61-62, Yokohama, Kanagawa, Japan, May 2012.

[5] B. Chen, J. Zhang, W. Xie, J. P. Jue, Y. Zhao, and G. Shen, "Cost-effective survivable virtual optical network mapping in flexible bandwidth optical networks," Journal of Lightwave Technology, vol. 34, no. 10, pp. 2398-2412, 2016.

[6] X. Xingsi and L. Jiawei, "A compact brain storm algorithm for matching ontologies," IEEE Access, vol. 8, pp. 43898-43907, 2020.

[7] B. Guo, C. Qiao, J. Wang et al., "Survivable virtual network design and embedding to survive a facility node failure," Journal of Lightwave Technology, vol. 32, no. 3, pp. 483-493, 2013.

[8] R. Lu and X. Nan, "Survivable multipath routing and spectrum allocation in OFDM-based flexible optical networks," Journal of Optical Communication and Network, vol. 5, no. 3, pp. 172-182, 2013.

[9] X. Xue and J. Chen, "Optimizing sensor ontology alignment through compact co-firefly algorithm," Sensors, vol. 20, no. 7, p. 2056, 2020.

[10] H. Kim, "Performance evaluation of revised virtual resources allocation scheme in network function virtualization (nfv) networks," Cluster Computing, vol. 22, no. S1, pp. 2331-2339, 2019.

[11] M. Chen, Y. Hao, M. Qiu, J. Song, D. Wu, and H. Iztok, "Mobility-aware caching and computation offloading in $5 \mathrm{~g}$ ultra-dense cellular networks," Sensors, vol. 16, no. 7, p. 974, 2016.

[12] L. Fang, X. Zhang, K. Sood, Y. Wang, and S. Yu, "Reliabilityaware virtual network function placement in carrier networks," Journal of Network \& Computer Applications, vol. 154, p. 102536, 2020.

[13] K. Odagiri, S. Shimizu, and N. Ishii, "Establishment of virtual policy based network management scheme by load experiments in virtual environment," International Journal of Computer Networks \& Communications, vol. 8, no. 3, pp. 181-194, 2016.

[14] R. J. Pfitscher, A. S. Jacobs, L. Zembruzki et al., "Guiltiness: a practical approach for quantifying virtual network functions performance," Computer Networks, vol. 161, pp. 14-31, 2019.

[15] S. I. Kuribayashi, "Virtual routing function deployment in nfv based networks under network delay constraints," International Journal of Computer Networks \& Communications, vol. 10, no. 1, pp. 35-44, 2018.

[16] A. D. Domenico, Y. F. Liu, and W. Yu, "Optimal virtual network function deployment for $5 \mathrm{~g}$ network slicing in a hybrid cloud infrastructure," IEEE Transactions on Wireless Communications, vol. 19, no. 12, pp. 7942-7956, 2020.

[17] R. Yu, G. Xue, V. T. Kilari, and X. Zhang, "Network function virtualization in the multi-tenant cloud," IEEE Network, vol. 29, no. 3, pp. 42-47, 2015.

[18] V. Malathi, S. Takehiro, B. Molly, R. Reza, O. Satoru, and Y. Naoaki, "Network function virtualization: a survey," IEICE Transactions on Communications, vol. E100.B, no. 11, pp. 1978-1991, 2017.

[19] K. Rakesh, M. Manoj, and A. K. Sarje, “A proactive load-aware gateway discovery in ad hoc networks for internet connectivity," International Journal of Computer Networks \& Communications, vol. 2, no. 5, pp. 275-282, 2010.

[20] Q. Zheng, R. Li, X. Li et al., "Virtual machine consolidated placement based on multi-objective biogeography- based 
optimization," Future Generation Computer Systems, vol. 54, pp. 95-122, 2016.

[21] D. Bhamare, A. Erbad, R. Jain, M. Zolanvari, and M. Samaka, "Efficient virtual network function placement strategies for cloud radio access networks," Computer Communications, vol. 127, pp. 50-60, 2018.

[22] V. Eramo, E. Miucci, M. Ammar, and F. G. Lavacca, "An approach for service function chain routing and virtual function network instance migration in network function virtualization architectures," IEEE/ACM Transactions on Networking, vol. 25, no. 4, pp. 2008-2025, 2017.

[23] T. Wen, H. Yu, G. Sun, and L. Liu, "Network function consolidation in service function chaining orchestration," in IEEE International Conference on Communications, Kuala Lumpur, Malaysia, 2016.

[24] A. Hameed, A. Khoshkbarforoushha, R. Ranjan et al., "A survey and taxonomy on energy efficient resource allocation techniques for cloud computing systems," Computing, vol. 98, no. 7, pp. 751-774, 2016.

[25] L. Qu, M. Khabbaz, and C. Assi, "Reliability-aware service chaining in carrier-grade softwarized networks," IEEE Journal on Selected Areas in Communications, vol. 36, no. 3, pp. 558573, 2018.

[26] A. N. Toosi, J. Son, Q. Chi, and R. Buyya, "Elasticsfc: autoscaling techniques for elastic service function chaining in network functions virtualization-based clouds," Journal of Systems and Software, vol. 152, pp. 108-119, 2019.

[27] L. Tang, G. Zhao, C. Wang, P. Zhao, and Q. Chen, "Queueaware reliable embedding algorithm for $5 \mathrm{~g}$ network slicing," Computer Networks, vol. 146, pp. 138-150, 2018.

[28] H. Xuan, Y. Wang, Z. Xu, S. Hao, and X. Wang, "Virtual optical network mapping and core allocation in elastic optical networks using multi-core fibers," Optics Communications, vol. 402, pp. 26-35, 2017.

[29] H. Xuan, S. Wei, Y. Feng, H. Guo, and Y. Li, “A new bi-level mathematical model and algorithm for vons mapping problem," IEEE Access, vol. 8, pp. 101797-101811, 2020.

[30] S. P. Singh and S. C. Sharma, "Implementation of a PSO based improved localization algorithm for wireless sensor networks," IETE Journal of Research, vol. 65, pp. 502-514, 2018.

[31] H. R. Tizhoosh, "Opposition-based learning: a new scheme for machine intelligence," in International Conference on International Conference on Computational Intelligence for Modelling, Control \& Automation, pp. 695-701, Vienna, Austria, Nov 2005.

[32] S. Hui, D. Zhi-Cheng, Z. Jia, W. Hui, and X. Hai-Hua, "Hybrid mean center opposition-based learning particle swarm optimization," Acta Electronica Sinica, vol. 47, no. 9, pp. 1809-1818, 2019.

[33] W. Fang, M. Zeng, X. Liu, W. Lu, and Z. Zhu, "Joint spectrum and it resource allocation for efficient vnf service chaining in inter-datacenter elastic optical networks," IEEE Communications Letters, vol. 20, no. 8, pp. 1539-1542, 2016.

[34] Q. Jiang, L. Wang, Y. Lin, X. Hei, and X. Lu, “An efficient multi-objective artificial raindrop algorithm and its application to dynamic optimization problems in chemical processes," Applied Soft Computing, vol. 58, no. 5, pp. 354-377, 2017. 